\title{
Raman Computational and Experimental Studies of Dopamine Detection
}

\author{
John D. Ciubuc ${ }^{1,2}$, Kevin E. Bennet ${ }^{3}$, Chao Qiu ${ }^{1}$, Matthew Alonzo ${ }^{1}$, William G. Durrer ${ }^{1}$ \\ and Felicia S. Manciu 1,2,4,* \\ 1 Department of Physics, University of Texas at El Paso, El Paso, TX 79968, USA; \\ jdciubuc@miners.utep.edu (J.D.C.); chaoqiu66@gmail.com (C.Q.); malonzo6@miners.utep.edu (M.A.); \\ wdurrer.utep.edu (W.G.D.) \\ 2 Department of Biomedical Engineering, University of Texas at El Paso, El Paso, TX 79968, USA \\ 3 Division of Engineering, Department of Neurologic Surgery, Mayo Clinic, Rochester, MN 55905, USA; \\ Bennet.Kevin@mayo.edu \\ 4 Border Biomedical Research Center, University of Texas at El Paso, El Paso, TX 79968, USA \\ * Correspondence: fsmanciu@utep.edu; Tel.: +1-915-747-8472
}

Received: 6 July 2017; Accepted: 25 September 2017; Published: 28 September 2017

\begin{abstract}
A combined theoretical and experimental analysis of dopamine (DA) is presented in this work with the objective of achieving more accurate detection and monitoring of this neurotransmitter at very low concentrations, specific to physiological levels. Surface-enhanced Raman spectroscopy on silver nanoparticles was employed for recording DA concentrations as low as $10^{-11}$ molar. Quantum chemical density functional calculations were carried out using Gaussian-09 analytical suite software. Relatively good agreement between the simulated and experimentally determined results indicates the presence of different DA molecular forms, such as uncharged $\mathrm{DA}^{ \pm}$, anionic $\mathrm{DA}^{-}$, and dopaminequinone. Disappearance of the strongest bands of dopamine around $750 \mathrm{~cm}^{-1}$ and $790 \mathrm{~cm}^{-1}$, which suggests its adsorption onto the metallic surface, is not only consistent with all of these DA configurations, but also provides additional information about the analyte's redox process and voltammetric detection. On the other hand, occurrence of the abovementioned Raman lines could indicate the formation of multilayers of DA or its presence in a cationic $\mathrm{DA}^{+}$form. Thus, through coordinated experiment and theory, valuable insights into changes observed in the vibrational signatures of this important neurotransmitter can be achieved for a better understanding of its detection at physiological levels, which is crucial if further optovoltammetric medical device development is envisioned.
\end{abstract}

Keywords: dopamine detection; label-free; surface-enhanced Raman spectroscopy; physiological levels; biosensors; computer simulation; silver nanocolloids

\section{Introduction}

Surface-enhanced Raman spectroscopy (SERS) was discovered over forty years ago through the enhancement of the Raman signal that was observed for pyridine adsorption on soft silver electrodes [1]. Since then, SERS has gone through multiple advances in understanding and expanding the technique. The SERS enhancement factor is now known to be due to two primary phenomena: electromagnetic (EM) and chemical (CM) enhancement mechanisms [2]. Electromagnetic enhancement serves as the bulk of the enhancement factor due to local EM field effects, providing enhancement to the order of $10^{4}-10^{10}[2,3]$. Chemical enhancement is due to metal-adsorbate charge resonance and interactions, and has a lower magnitude influence, contributing a factor of $10^{2}-10^{3}$ [2-4]. The level of enhancement that can be achieved in SERS depends, of course, on the nature of the metallic nanoparticles themselves. Furthermore, adsorbates at specific locations in the proximity of the nanoparticles exhibit Raman 
'hot-spot' effects; such locations are SERS-active sites, at which an impressive gain in the amplification of the Raman signal is observed $[2,5,6]$. SERS has even led to the study of single molecules, achieving an enhancement factor of over $10^{10}$ through the adhesion of nanoparticles to individual molecules [5-8].

Dopamine (DA) is the focus of the SERS study presented in this paper. It is one of the most studied neurotransmitters, as it is centrally involved in many neurological mechanisms, ranging from those of the extrapyramidal motor tracts of the central nervous system to that of psychostimulant addiction [9-13]. As such, in addition to the relevance of detecting it accurately, it has also been used as a potent drug for activating receptors in the brain, heart muscle, kidney, and gut [9-13]. The level of receptor activation strongly depends on the dose administrated. The need for its accurate detection at physiological levels is largely the reason for the development of various techniques, ranging from electrochemistry [14], chromatography [15], and gravimetry [16,17], to optical spectroscopy [5-8]. From a chemical perspective, its ionizable functional groups-an ethyl ammonium, which acts as a proton acceptor, and two phenolic hydroxyls, which act as donors-can participate in DA molecular bonding. At physiological levels, the dominant presence of ionized DA has been reported from both experimental $[14,15,18]$ and theoretical $[16,17,19]$ results.

Besides the degree of adsorption and polarizability of a particular analyte, another factor that is relevant to our work here, because it can significantly influence the SERS signal, is analyte orientation in the vicinity of the metallic nanoparticles $[5,6,8,19]$. Under its influence, SERS data exhibit unique characteristics, commonly showing rapid changes in vibrational intensities. In addition, analyte-nanoparticle orientation with respect to the Raman excitation source can definitely affect the outcome of such a measurement. As a result, in some cases, the relative Raman intensities of some vibrational lines can be completely suppressed. For example, there is no observable activity of DA vibrations at 750 and $795 \mathrm{~cm}^{-1}$, vibrations associated with either the in-plane phenolic ring bending mode or with the out-of-plane O-H and C-H bending modes $[19,20]$. On the other hand, if DA orientation is optimum, remarkable increases in Raman signatures are obtained. Usually, vibrations associated with larger components of polarizability in the direction normal to the silver nanoparticle surface are enhanced more.

The aid of computational methods and powerful computers currently offers considerable advantages in identifying structural modifications of analytes and their consequent influence on vibrational outcomes. There is a general consensus that density functional theory (DFT) provides sufficiently consistent results that are in relatively good agreement with experimentally determined molecular frequencies. Thus, prediction of DA orientations and structural modifications through computational analysis allows for an effective understanding of the resulting Raman spectrum.

Although this study mainly aims to make valuable predictions of Raman intensities and wavenumber locations through combinatorial computational and experimental analysis, such evaluations are of importance for understanding other physicochemical phenomena resulting from currently employed fast-scan cyclic voltammetric devices. In this context, it is worth mentioning that roughened electrode surfaces have previously been used as SERS substrates [1,21,22]. Moreover, the current Raman evaluations are, in fact, based on experimental results of dopamine recordings for as low as $10^{-11}$ molar. The ultrasensitivity of the experimental data, which are definitely at the normal physiological levels of DA, is another indication of the value of the results discussed in this work.

\section{Materials and Methods}

\subsection{Materials and Equipment}

Silver nitrate $\left(\mathrm{AgNO}_{3},>99 \%\right)$, sodium borohydride $\left(\mathrm{NaBH}_{4},>99 \%\right)$, and citric acid trisodium salt dihydrate $\left(\mathrm{C}_{6} \mathrm{H}_{5} \mathrm{Na}_{3} \mathrm{O}_{7} \cdot 2 \mathrm{H}_{2} \mathrm{O}, 99 \%\right)$ reagents purchased from Sigma-Aldrich and ACROS, respectively, were used for the synthesis of silver nanoparticles (Ag NPs) following a previously reported procedure [23]. Briefly, the chemical process consists of mixing $20 \mathrm{~mL}$ of $1 \%(w / v)$ citrate solution with $75 \mathrm{~mL}$ of ultrapure water and heating the solution to $80{ }^{\circ} \mathrm{C}$ until stable. Next, $1.7 \mathrm{~mL}$ of $1 \%$ 
$(w / v) \mathrm{AgNO}_{3}$ and $2 \mathrm{~mL}$ of $0.1 \%(w / v)$ freshly prepared $\mathrm{NaBH}_{4}$ solutions were added to the mixture. The reaction solution was kept at $80{ }^{\circ} \mathrm{C}$ under vigorous stirring for $30 \mathrm{~min}$ and cooled to room temperature. Purification of the resulting solution to remove the excess of organic and unreacted impurities was then performed several times; it consisted of washing with ultrapure water and centrifugation. Next, $90 \mu \mathrm{L}$ of synthesized Ag NPs was mixed with $10 \mu \mathrm{L}$ a $10^{-10}$ molar solution of DA. The mixed solutions were sonicated for $20 \mathrm{~s}$, then drop-cast on clean cover slips to form dense, uniform Ag NP thin films.

The Raman measurements were acquired at ambient conditions in a backscattering geometry with an alpha 300RAS WITec system (WITec GmbH, Ulm, Germany). A 532-nm excitation of a frequency-doubled neodymium-doped yttrium-aluminum-garnet (Nd:YAG) laser that was restricted to a power output of about $100 \mu \mathrm{W}$ to avoid sample damage and a $20 \times$ objective (Olympus, Tokyo, Japan) were used. Time series Raman spectra, each of 200 milliseconds, were recorded. WITec Control 1.60 software was employed for fast data acquisition.

\subsection{Computational Analysis}

The quantum chemical density functional calculations were carried out using Gaussian-09 analytical suite software. The DA molecule was first energetically optimized for all of its forms, neutral $\left(\mathrm{DA}^{0}\right)$, anionic $\left(\mathrm{DA}^{-}\right)$, and cationic $\left(\mathrm{DA}^{+}\right)$, and their vibrational frequencies were computed using the Becke three hybrid exchange [24] and Lee-Yang-Parr correlation functional [25], B3LYP. A Pople split valence diffused and polarized $6-311++G(d, p)$ basis set was used for such calculations. The Raman activities, $S_{i}$, obtained from the Gaussian- 09 software were further converted into relative Raman intensities $\left(I_{i}\right)$ using the following equation [26-28]:

$$
I_{i}=\frac{f S_{i}\left(v_{0}-v_{i}\right)^{4}}{v_{i}\left[1-\exp \left(-\frac{h c v_{i}}{k T}\right)\right]}
$$

where $v_{0}$ is the excitation frequency $\left(532 \mathrm{~nm}=18,796.99 \mathrm{~cm}^{-1}\right), v_{i}$ is the vibrational wavenumber of the $i^{\text {th }}$ normal mode, $h$ is the Planck's constant, $c$ is the speed of light, and $k$ is the Boltzmann constant. A normalization factor $f=1 \mathrm{e}^{-12}$ was used for all peak intensities and a value of 293.15 Kelvin was used for the temperature, $T$. Lorentzian band shapes were also applied to the resulting Raman intensity spectrum, using a full width at half maximum (FWHM) of $7 \mathrm{~cm}^{-1}$. To enable data plotting, parsing of the Gaussian-09 Raman output data, which was completed through an in-house algorithm developed in the $\mathrm{C}++$ language utilizing the $\mathrm{Qt}$ framework, as well as conversion through MATLAB version r2016a, was performed.

\section{Results}

Group theory predicts that both planar and non-planar species are expected in all of the DA forms (i.e., $\mathrm{DA}^{0}, \mathrm{DA}^{-}$, and $\mathrm{DA}^{+}$), which all have $\mathrm{C}_{\mathrm{S}}$ point group symmetry $[19,26]$. Furthermore, both species are Raman and infrared (IR) active. For Raman in particular, the polarized bands are mainly associated with in-plane vibrations and the depolarized ones with out-of-plane vibrations.

The optimized molecular structure of $\mathrm{DA}^{0}$ and the calculated Raman vibrations using the Gaussian software are presented in Figure 1A,B, respectively. We also show in Figure 1C the experimental Raman spectrum of solid dopamine (i.e., of DA powder), for easier comparison between measurements and simulations. There is relatively good agreement between the peaks of the theoretically estimated frequencies, which have been scaled by a factor of 0.98 , and the observed vibrations, except for some variations in the peak intensities. Typically, scaling factors have been used in the literature to overcome systematic empirical errors originating from the force field constants employed in quantum mechanical approaches [19,26]. Although it is a debated subject [29], as a single uniform scaling factor (or frequency scaling) cannot entirely account for all errors, we still consider it in this work, as our perspective stems from a desire to obtain a reliable understanding of vibrational assignments as compared with 
experimentally observed phenomena. Even higher level theoretical calculations often cannot perfectly account for the complexity of experimental specifics in their entirety.

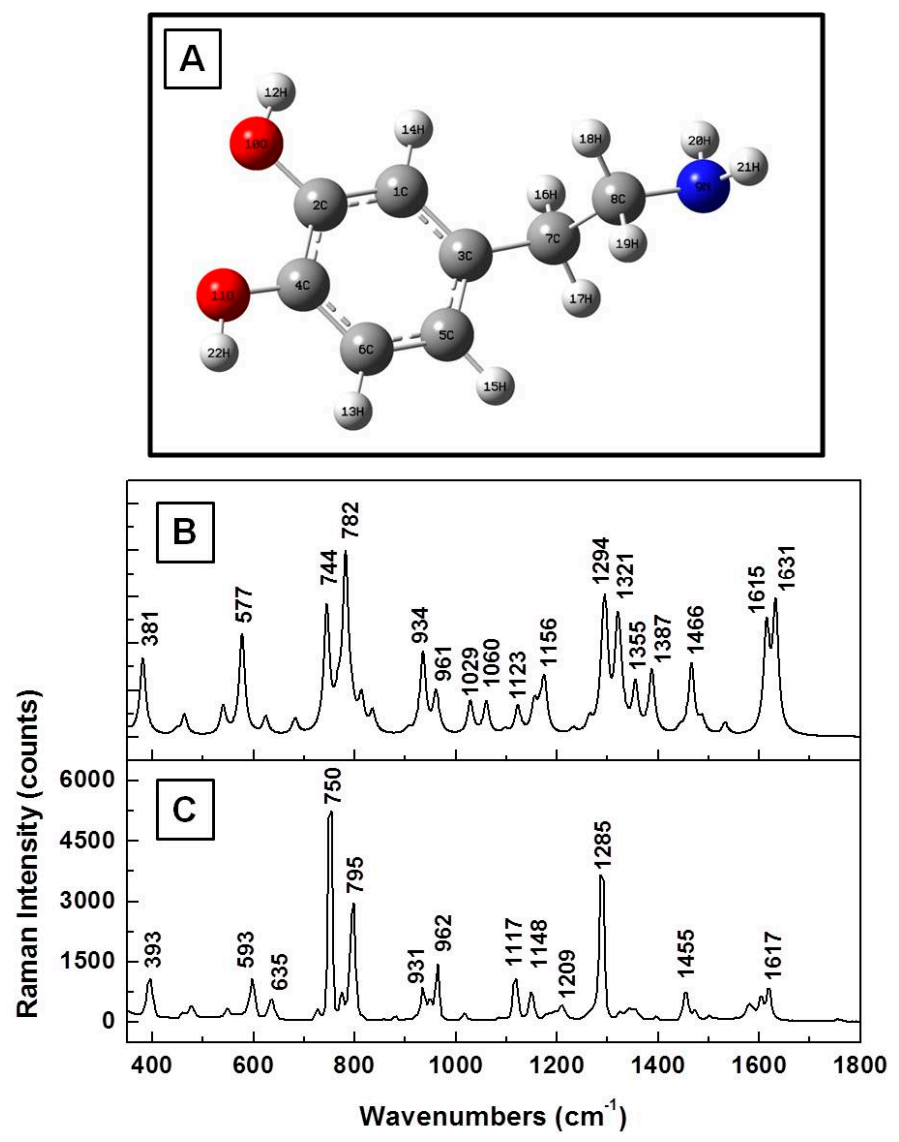

Figure 1. (A) Dopamine structural representation in neutral state, $\mathrm{DA}^{0}$. Red and blue colors were used for oxygen and nitrogen atoms, respectively; $(\mathbf{B}, \mathbf{C})$ Theoretically calculated and experimentally measured Raman vibrations of dopamine, respectively. The Raman spectrum was recorded for the standard dopamine powder.

Although similar calculated frequencies for DA have been reported in the literature [19], some repetition of results in research is inevitable and contributes to such supportive purposes as cross-checking and confirmation of reproducibility. At a glance, there are discrepancies of $10 \pm 2 \mathrm{~cm}^{-1}$, on average, between the experimentally determined and estimated values. For the unscaled frequencies (data not reported here), these discrepancies become larger at higher frequency, indeed suggesting an overestimation of the force field constant used in Gaussian simulations. The measured and computationally estimated values of the main vibrational modes along with their assignments are summarized in Table 1 . The calculated values presented include a scaling factor of 0.98 . The assignments were made by direct visualization with Gaussview software, as well as by comparison with the literature [28-31].

Based on previous studies, the deprotonation of dopamine is expected when it is dissolved in water, which can result in the molecular transformation of DA into dopaminequinone, besides its other forms mentioned above (e.g., $\mathrm{DA}^{-}$, and $\left.\mathrm{DA}^{+}\right)[19,32-34]$. Since this process is likely to occur in the preparation of our $10^{-11} \mathrm{M}$ dopamine solution, we schematically present it in Figure 2, together with molecular representations of the two compounds. The quinonic form is considered first due to the obvious symmetry of the DA molecule as regards the $\mathrm{O}-\mathrm{H}$ and $\mathrm{N}-\mathrm{H}$ bonds, which is known to result in a one-step redox reaction involving two electrons and two protons. The anionic and cationic forms are also investigated in this work. 


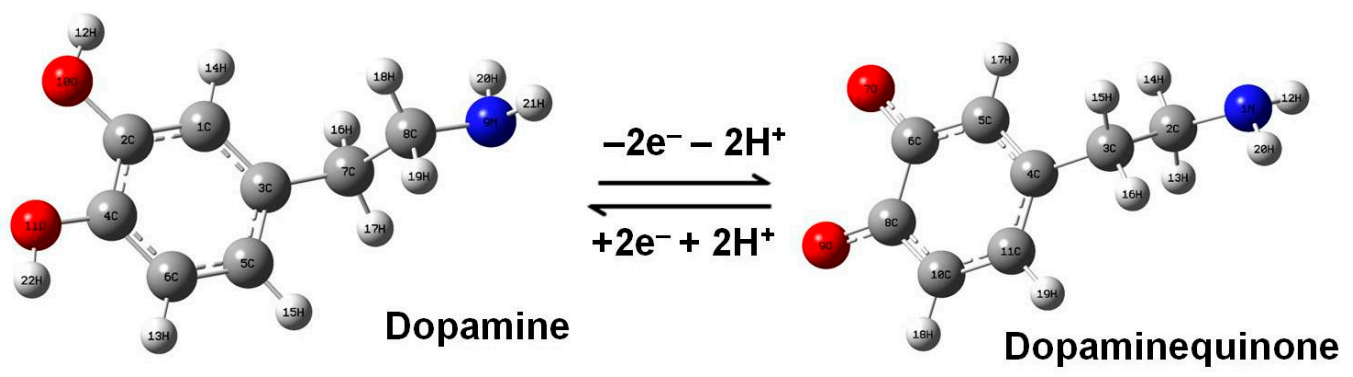

Figure 2. The redox process of dopamine consisting of the transfer of two electrons and two protons. The structural representations of neutral dopamine and dopaminequinone are also presented and appropriately labeled.

Table 1. Theoretically calculated and experimentally measured Raman vibrations of dopamine with their tentative assignments.

\begin{tabular}{ccc}
\hline Calculated $\left.\mathbf{( c m}^{-\mathbf{1}}\right)$ & Measured $\left.\mathbf{( c m}^{-\mathbf{1}}\right)$ & Assignment \\
\hline 381 & 393 & CH wagging, ring deformation \\
577 & 593 & CH in-plane ring deformation \\
622 & 635 & CH wagging; aliphatic chain C-C vibrations \\
744 & 750 & CH out-of-plane; ring deformation (two band response) \\
782 & 795 & CH out-of-plane; ring deformation (two band response) \\
934 & 931 & NH twisting \\
961 & 962 & NH twisting; CH wagging; ring deformation \\
1029 & & CH wagging \\
1060 & 1117 & C-C-N stretching; CH wagging \\
1123 & 1148 & CH twisting; NH twisting; CN stretching \\
1156 & 1209 & OH rocking; CH aromatic rocking; weak ring breathing; $\mathrm{CH}$ wagging \\
1294 & 1285 & CO stretching \\
1321 & & Ring breathing; CH aromatic rocking; CH twisting \\
1355 & & Ring breathing; CH aromatic in-plane rocking; CH twisting \\
1387 & Ring deformation; OH scissoring; CH twisting \\
1466 & 1455 & CH wagging; NH twisting \\
1615 & 1617 & CH scissoring \\
1631 & & Ring deformation, OH scissoring \\
1634 & & NH $H_{2}$ scissoring \\
& & Benzene ring deformation \\
\hline
\end{tabular}

The effect of a SERS environment on the Raman vibrational frequencies of dopaminequinone and neutral $\mathrm{DA}^{0}$ is further analyzed from theoretical and experimental perspectives in Figure 3A-D. The structural representations of the analyte in the proximity of silver after energy optimization using an LanL2DZ basic set that takes into consideration the pseudopotentials for metal atoms are shown in Figure 3A,B. A comparison of the estimated Raman frequencies of dopaminequinone and the Raman results that were obtained experimentally, which are presented in Figure 3C, not only shows good agreement, but, more importantly, confirms the disappearance of dominant DA Raman vibrations at $750 \mathrm{~cm}^{-1}$ and $795 \mathrm{~cm}^{-1}$, in both the simulation and the experimental data. However, this disappearance does not occur for the calculated vibrational lines of neutral $\mathrm{DA}^{0}$ in the proximity of Ag NPs, as observed in Figure 3D. On the contrary, they are the most intense Raman features seen in this figure, besides two other vibrations around $550 \mathrm{~cm}^{-1}$ and $580 \mathrm{~cm}^{-1}$. The stronger interaction of the dopaminequinonic form with silver could be attributed to a somewhat greater electronegativity of oxygen atoms in this configuration than in $\mathrm{DA}^{0}$. Dominant Raman vibrations around $1360 \mathrm{~cm}^{-1}$ and $1510 \mathrm{~cm}^{-1}$ are observed in Figure 3C for dopaminequinone interaction with Ag NPs. 

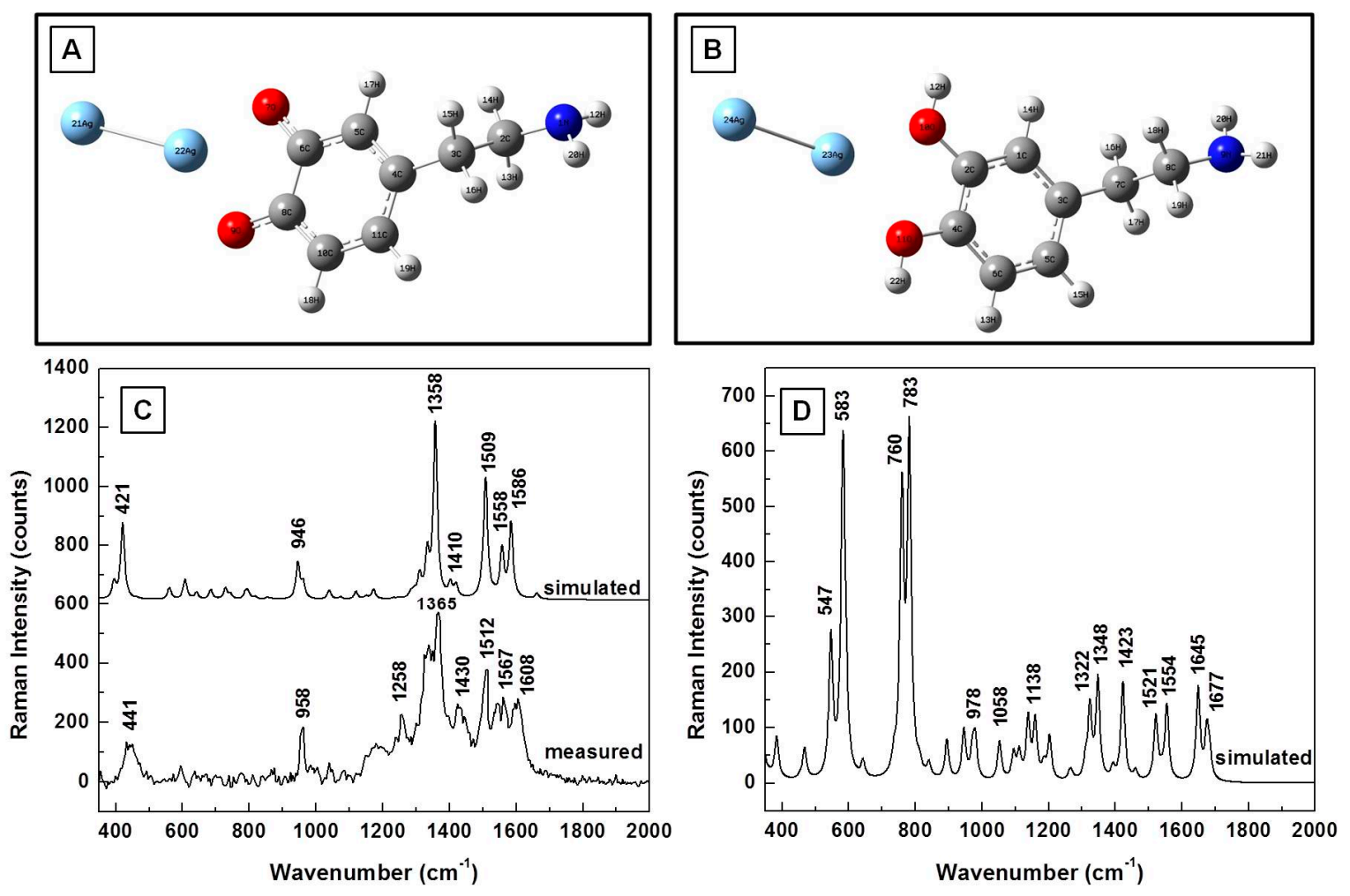

Figure 3. (A,B) Dopaminequinone and neutral $\mathrm{DA}^{0}$ structural representations in the proximity of a silver dimer after energy optimization, respectively; (C) Theoretically estimated and experimentally recorded Raman vibrational spectra of dopaminequinone; (D) Theoretically calculated Raman spectrum of dopamine in the proximity of silver.

In view of the fact that other SERS vibrational lines have been experimentally observed and reported in the literature for dopamine, and the dissociation of the $\mathrm{DA}^{0}$ molecule into $\mathrm{DA}^{-}$and $\mathrm{DA}^{+}$in water is also expected during sample preparation, the analysis of these ionic DA forms alone and in the presence of silver is presented in Figure 4A-D and Figure 5A-D. The energetically optimized structural representations of these anionic and cationic forms are shown in Figure 4A,B and in Figure 5A,B, respectively. Slight deformations (torsional and bending) of the amine chains are evident in both cases. Furthermore, while the silver dimer has a planar orientation in regard to the benzene ring and is asymmetrically closer to the oxygen in the anionic case, a quasi-planar position of the silver towards the primary ammonium $\mathrm{NH}_{3}{ }^{+}$functional group is seen for the cationic form.

The simulated Raman vibrational frequencies in the regions of interest for $\mathrm{DA}^{-}$and $\mathrm{DA}^{+}$, which are presented in Figures $4 \mathrm{C}$ and $5 \mathrm{C}$, respectively, show dominant peaks around 640, 750, 780, 930, $1150,1280,1530$, and $1610 \mathrm{~cm}^{-1}$ for the anionic form and around 580, 800, 820, 1180, 1330, 1390, 1480 , and $1640 \mathrm{~cm}^{-1}$ for the cationic form. The silver influence in the computed Raman vibrational lines, which is shown in Figures $4 \mathrm{D}$ and 5D, reveals a decrease in the intensities of DA characteristic lines around $750 \mathrm{~cm}^{-1}$ and $780 \mathrm{~cm}^{-1}$ in the anionic case. The opposite behavior is observed for $\mathrm{DA}^{+}$, resembling more that of $\mathrm{DA}^{0}$. Small variations in the positions and intensities of other Raman lines are also seen in these figures. For a comparative analysis, an experimental Raman spectrum is also presented in Figure 4D. The spectra in this figure are again vertically translated for easier visualization and appropriately labeled.

An interesting observation concerns the computed line around $1150 \mathrm{~cm}^{-1}$, which has been previously measured $[19,21]$. The current results seem to indicate that it originates from in-plane bending of the anionic form of dopamine. This assumption better supports the notion of its presence in aqueous solution; however, during the drying process of the current sample preparation, some water 
molecules might remain attached to dopamine ions, and therefore not evaporate. As such, they could provide screening, hindering the re-association and neutralization of the charges. This screening could be quite strong, as it is proportional to the high dielectric constant of water (i.e., $\varepsilon=80$ ). Another potential explanation is the creation of image charges in close proximity to the metallic Ag NPs, which can decrease the Born energy to a lower value than that of the entropy related to the dissociation of charges. As such, when the value of the entropy is large, the DA molecules remain charged and a dynamic equilibrium between the cationic and anionic forms of the dopamine molecules remains. Thermal energy acquired due to laser excitation could also be a contributing factor. Although we would not eliminate this possibility, we consider it less likely, as the laser power of $100 \mu \mathrm{W}$ remained constant for all measurements.

Charge redistribution and formation of intramolecular hydrogen bonds is also possible. Such an uncharged $\mathrm{DA}^{ \pm}$structure in the vicinity of the silver dimer is presented in Figure $6 \mathrm{~A}$, and is computationally and experimentally analyzed in Figure 6B. An obvious bending of the amine chain towards the silver dimer is observed in Figure 6A, with the dimer oriented quasi-perpendicularly to the benzene ring of the molecule. More notably, this energetically stable configuration suggests that dopamine could interact with the silver surface through both functional groups: the known proton donor hydroxyl group and the $\mathrm{NH}_{3}{ }^{+}$. Besides the SERS cases of dopaminequinonic and anionic forms, where no DA characteristic vibrations around $750 \mathrm{~cm}^{-1}$ and $790 \mathrm{~cm}^{-1}$ or their very slight presence were computationally obtained and experimentally confirmed, only their weak contributions are theoretically predicted in Figure $6 \mathrm{~B}$ for this $\mathrm{DA}^{ \pm}$configuration.
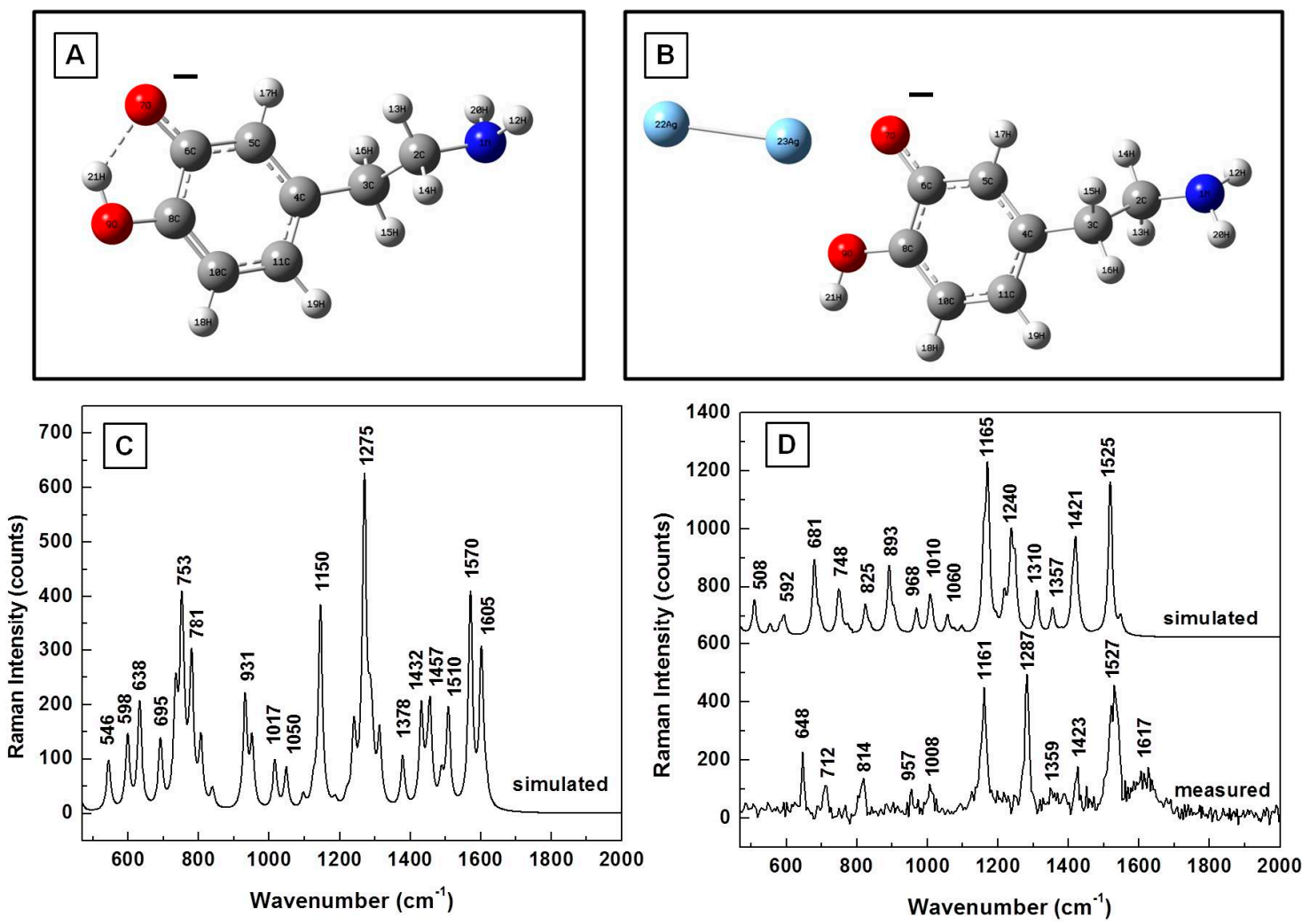

Figure 4. (A,B) Structural representations of the anionic form of dopamine without and with the silver dimer, respectively; (C) Theoretically calculated Raman vibrations of $\mathrm{DA}^{-}$; and (D) Theoretically calculated and experimentally measured Raman vibrations of the anionic form in the proximity of silver. The spectra are vertically translated for easier visualization and appropriately labeled. 

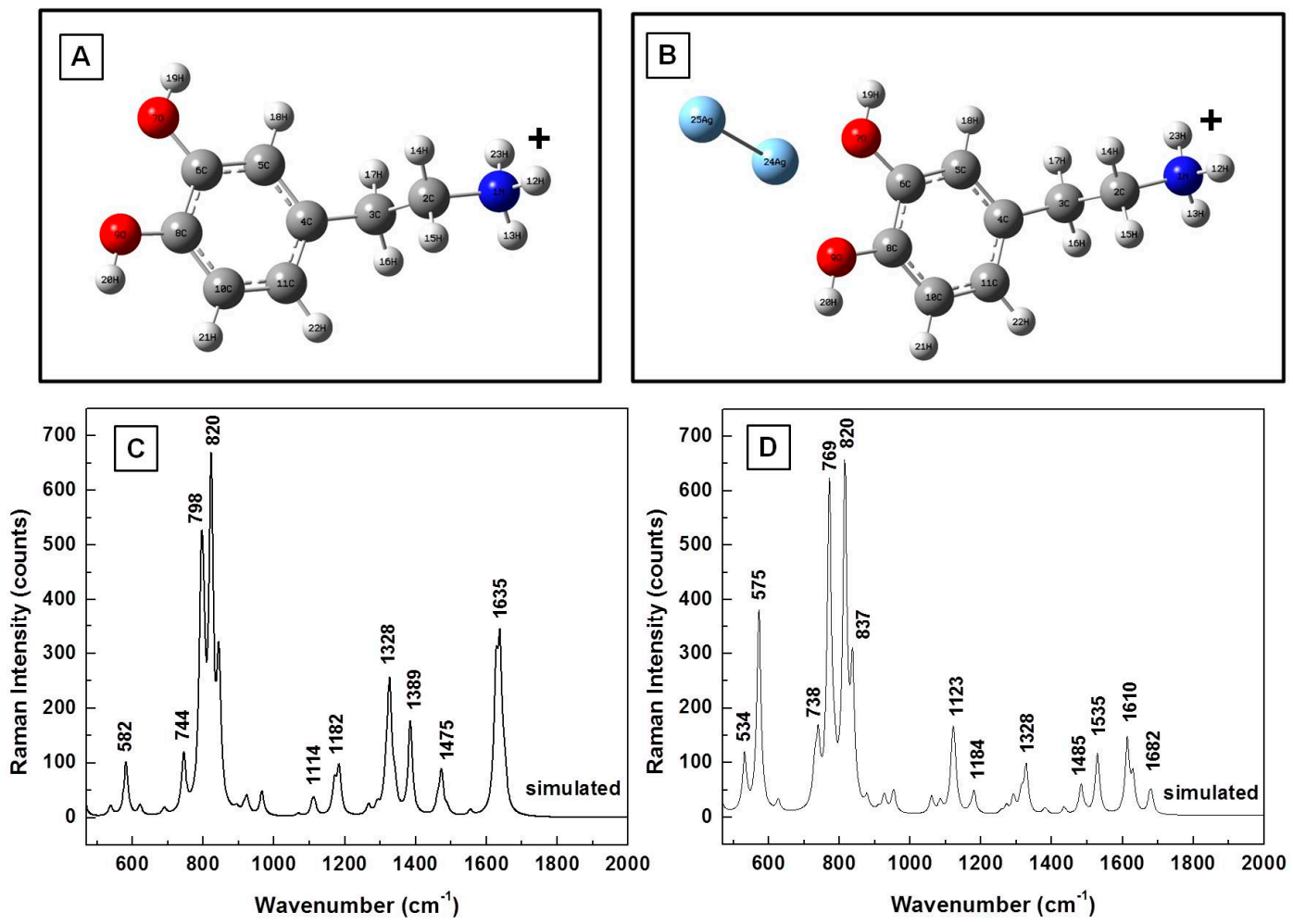

Figure 5. (A,B) Structural representations of the cationic form of dopamine without and with the silver dimer, respectively; (C,D) Theoretically calculated Raman vibrations of $\mathrm{DA}^{+}$alone and in the proximity of silver, respectively.

The relatively good agreement between the calculated and measured results, which are also presented in Figure 6B, not only confirms the existence of this configuration, but also provides additional insights into DA morphological changes that might occur during analyte detection at physiological levels by other techniques such as electrochemical fast-scan cyclic voltammetry [1,21,22]. The current results suggest that while dopaminequinone is more likely detected electrochemically as the analyte's oxidized form, when the potential is applied in the negative direction, the $\mathrm{DA}^{ \pm}$ configuration is more probably detected as the reduced form. Furthermore, it is obvious that the analyte undergoes reorientation and deformation when adsorbed onto the silver surface, from a quasi-planar, vertically oriented structure with the oxygen atoms as direct adsorption sites (see dopaminequinone form in Figure 3B) to a quite contorted, bent structure with oxygen and nitrogen as adsorption sites (see DA ${ }^{ \pm}$form in Figure 6A).

Characteristic signatures corresponding to each DA configuration can also be detected, such as the vibration lines around 950 and $1360 \mathrm{~cm}^{-1}$ for dopaminequinine, around 1160,1290, and $1530 \mathrm{~cm}^{-1}$ for the anionic form, and around 1280 and $1640 \mathrm{~cm}^{-1}$ for $\mathrm{DA}^{ \pm}$. In order to eliminate the random fluctuations expected in any experimental data, and for better comprehension of the likelihood of occurrence for these configurations, we present in Figure 7 the overall average of 400 SERS spectra, which were collected in different spots on the sample (eight different time series acquisitions of 50 SERS spectra each, at 200 milliseconds per spectrum). Considering the intensities of the vibrational lines observed in this spectrum, the dopaminequinone form appears to be the predominant configuration, followed by that of the anionic form, and that of the $\mathrm{DA}^{ \pm}$form; the latter two forms might occur with approximately the same proportions. However, due to the similarity in some of their signatures, such as the $1290 \mathrm{~cm}^{-1}$ line for the anionic form and the $1280 \mathrm{~cm}^{-1}$ vibration for the $\mathrm{DA}^{ \pm}$form, together with the typical variation in full width at half maximum (FWHM) of the recorded Raman features, it is difficult to determine a reliable numerical estimate of their contributions. In the context of this 
study, it is also worth mentioning that none of the characteristic neutral $\mathrm{DA}^{0}$ (standard DA powder, see Figure 1) signatures around 750 and $790 \mathrm{~cm}^{-1}$ were detected experimentally, as observed in this spectrum. Their occurrence would imply the potential presence of multilayer neutral $\mathrm{DA}^{0}$, or of cationic $\mathrm{DA}^{+}$.

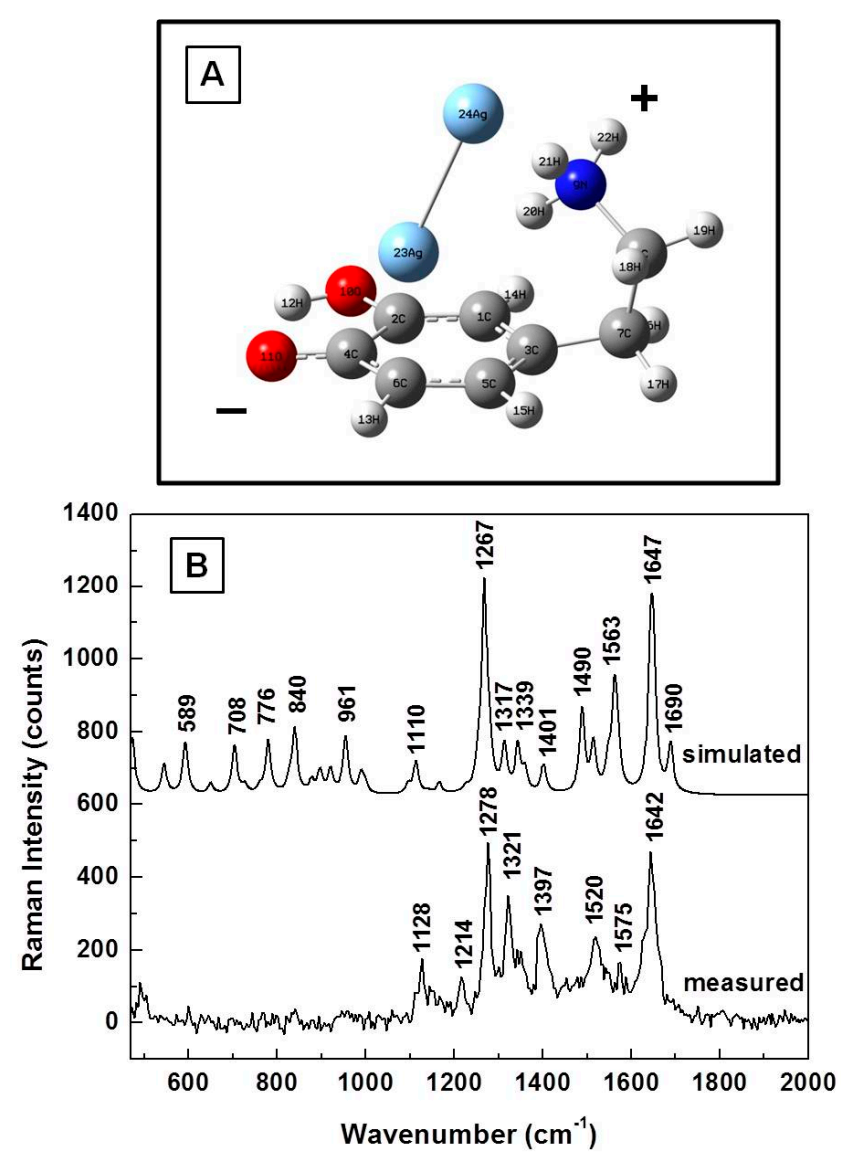

Figure 6. (A) Dopamine $\mathrm{DA}^{ \pm}$structural representation in the proximity of silver; (B) Theoretically calculated and experimentally measured Raman vibrations of $\mathrm{DA}^{ \pm}$, as labeled. The spectra are vertically translated for easier visualization.

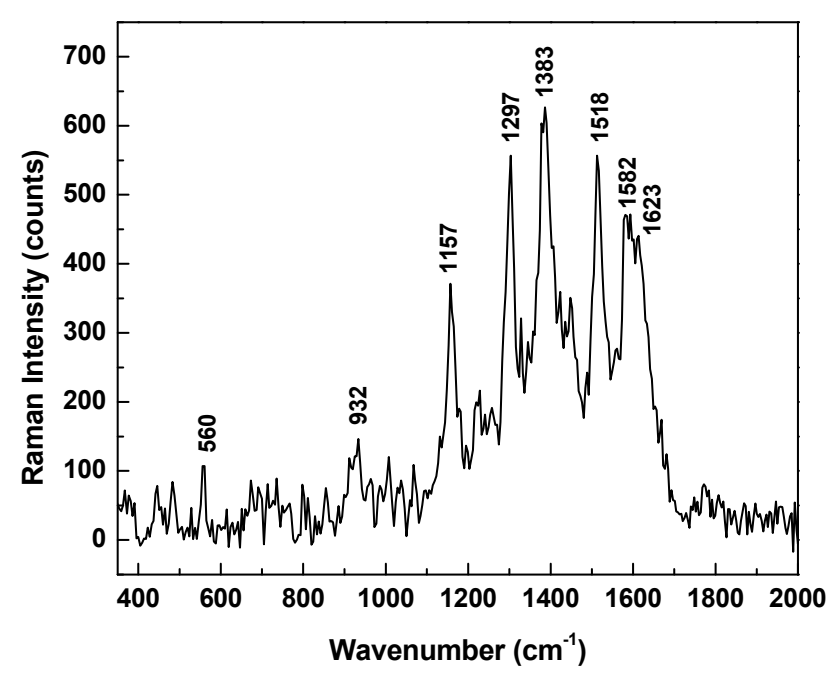

Figure 7. Overall average of 400 SERS spectra recorded in different spots on the sample (eight different time series acquisitions of 50 SERS spectra each, at 200 milliseconds per spectrum). 


\section{Conclusions}

To conclude, in this SERS study we present a comparative theoretical and experimental approach to the detection of dopamine at a concentration of $10^{-11}$ molar, which is definitely lower than normal physiological levels. Although the current measurements were not performed in aqueous solution, they facilitate a reliable understanding of vibrational assignments. Not only is the usual broadening of Raman bands that is characteristic of measurements in liquids eliminated in this case, but since the consideration of aqueous media would further complicate computational analysis, we have simplified the comparison and assessment of results. An even greater experimental challenge would be the question of obtaining a common consensus on the reproducibility of Raman intensities in a liquid environment. Especially at low concentrations, this issue involves the probability that molecules will become localized in regions of intense optical fields trapped between adjacent plasmonic surfaces, and thereby be subjected to SERS enhancement of their inherently weak Raman signals. By eliminating such limitations, we were able to acquire important insights into the complexity of dopamine detection at a very low concentration.

The relatively good agreement between the simulated and experimentally determined results suggests the possibility that different forms of the DA molecule, such as uncharged $\mathrm{DA}^{ \pm}$, anionic DA $^{-}$, as well as dopaminequinone, are present. All of these molecular configurations, in a SERS environment, also lose the dominant DA Raman lines at $750 \mathrm{~cm}^{-1}$ and $790 \mathrm{~cm}^{-1}$, suggesting their adsorption onto the metallic surface under different orientations. The occurrence of these features would imply the potential presence of multilayer neutral $\mathrm{DA}^{0}$, or that of cationic $\mathrm{DA}^{+}$. From a redox reaction perspective, which is known to take place in electrochemical measurements, the dopaminequinone form is more likely detected during the oxidation process, while the $\mathrm{DA}^{ \pm}$form more probably occurrs during reduction. The ultrasensitivity of the current experimental data, in combination with the theoretical analysis presented in this work, definitely provides valuable information for the advancement of the detection and monitoring of dopamine.

Acknowledgments: This work was supported by the NIH U01 NS090455 award, the NIH NIMHHD 2G12MD007592 award, The Grainger Foundation, and by a research agreement between the University of Texas at El Paso and the Mayo Clinic.

Author Contributions: Felicia S. Manciu conceived the idea for the study, its coordination, data analysis, and the draft of the manuscript. John D. Ciubuc, William G. Durrer, and Kevin E. Bennet contributed to data analysis and helped to draft the manuscript. John D. Ciubuc, Matthew Alonzo, and Chao Qiu contributed to producing theoretical Raman data, acquiring experimental Raman data, and sample preparation, respectively. All authors contributed significant effort to the manuscript preparation.

Conflicts of Interest: The authors declare no conflict of interest.

\section{References}

1. Fleischmann, M.; Hendra, P.J.J.; McQuillan, A.J.J. Raman spectra of pyridine adsorbed at a silver electrode. Chem. Phys. Lett. 1974, 26, 163-166. [CrossRef]

2. Dieringer, J.A.; Mcfarland, A.D.; Shah, N.C.; Stuart, D.A.; Whitney, A.V.; Yonzon, C.R.; Young, M.A.; Zhang, X.; Van Duyne, R.P. Introductory Lecture Surface enhanced Raman spectroscopy: New materials, concepts, characterization tools, and applications. Faraday Discuss. 2006, 132, 9-26. [CrossRef] [PubMed]

3. Virga, A.; Rivolo, P.; Frascella, F.; Angelini, A.; Descrovi, E.; Geobaldo, F.; Giorgis, F. Silver Nanoparticles on Porous Silicon: Approaching Single Molecule Detection in Resonant SERS Regime. J. Phys. Chem. C 2013, 117, 20139-20145. [CrossRef]

4. Otto, A. The 'chemical' (electronic) contribution to surface-enhanced Raman scattering. J. Raman Spectrosc. 2005, 36, 497-509. [CrossRef]

5. Qian, X.-M.; Nie, S.M. Single-molecule and single-nanoparticle SERS: From fundamental mechanisms to biomedical applications. Chem. Soc. Rev. 2008, 37, 912-920. [CrossRef] [PubMed]

6. Stranahan, S.M.; Willets, K.A. Super-resolution optical imaging of single-molecule SERS hot spots. Nano Lett. 2010, 10, 3777-3784. [CrossRef] [PubMed] 
7. Qiu, C.; Bennet, K.E.; Tomshine, J.R.; Hara, S.; Ciubuc, J.D.; Schmidt, U.; Durrer, W.G.; McIntosh, M.B.; Eastman, M.; Manciu, F.S. Ultrasensitive Detection of Neurotransmitters by Surface Enhanced Raman Spectroscopy for Biosensing Applications. Bionterface Res. Appl. Chem. 2017, 1, 1921-1926.

8. Le Ru, E.C.; Meyer, M.; Etchegoin, P.G. Proof of single-molecule sensitivity in Surface Enhanced Raman Scattering (SERS) by means of a two-analyte technique. J. Phys. Chem. B 2006, 110, 1944-1948. [CrossRef] [PubMed]

9. Zhang, A.; Neumeyer, J.L.; Baldessarini, R.J. Recent progress in development of dopamine receptor subtype-selective agents: Potential therapeutics for neurological and psychiatric disorders. Chem. Rev. 2007, 107, 274-302. [CrossRef] [PubMed]

10. De la Fuente-Fernández, R.; Ruth, T.J.; Sossi, V.; Schulzer, M.; Calne, D.B.; Stoessl, A.J. Expectation and dopamine release: Mechanism of the placebo effect in Parkinson's disease. Science 2001, 293, 1164-1166. [CrossRef] [PubMed]

11. Stewart, A.J.; Hendry, J.; Dennany, L. Whole Blood Electrochemiluminescent Detection of Dopamine. Anal. Chem. 2015, 87, 11847-11853. [CrossRef] [PubMed]

12. Meister, B. Neurotransmitters in key neurons of the hypothalamus that regulate feeding behavior and body weight. Physiol. Behav. 2007, 92, 263-271. [CrossRef] [PubMed]

13. Bennet, K.E.; Tomshine, J.R.; Min, H.-K.; Manciu, F.S.; Marsh, M.P.; Paek, S.B.; Settell, M.L.; Nicolai, E.N.; Blaha, C.D.; Kouzani, A.Z.; et al. A Diamond-Based Electrode for Detection of Neurochemicals in the Human Brain. Front. Hum. Neurosci. 2016, 10, 102-112. [CrossRef] [PubMed]

14. Li, H.; Wang, X.; Yu, Z. Electrochemical biosensor for sensitively simultaneous determination of dopamine, uric acid, guanine, and adenosine based on poly-melamine and nano Ag hybridized film-modified electrode. J. Solid State Electrochem. 2014, 18, 105-113. [CrossRef]

15. Heidbreder, C.A.; Lacroix, L.; Atkins, A.R.; Organ, A.J.; Murray, S.; West, A.; Shah, A.J. Development and application of a sensitive high performance ion-exchange chromatography method for the simultaneous measurement of dopamine, 5-hydroxytryptamine and norepinephrine in microdialysates from the rat brain. J. Neurosci. Methods 2001, 112, 135-144. [CrossRef]

16. Fourati, N.; Seydou, M.; Zerrouki, C.; Singh, A.; Samanta, S.; Maurel, F.; Aswal, D.K.; Chehimi, M. Ultrasensitive and Selective Detection of Dopamine Using Cobalt-Phthalocyanine Nanopillar-Based Surface Acoustic Wave Sensor. ACS Appl. Mater. Interfaces 2014, 6, 22378-22386. [CrossRef] [PubMed]

17. Maouche, N.; Ktari, N.; Bakas, I.; Fourati, N.; Zerrouki, C.; Seydou, M.; Maurel, F.; Chehimi, M.M. A surface acoustic wave sensor functionalized with a polypyrrole molecularly imprinted polymer for selective dopamine detection. J. Mol. Recognit. 2015, 28, 667-678. [CrossRef] [PubMed]

18. Meulemans, A.; Poulain, B.; Baux, G.; Tauc, L.; Henzel, D. Micro carbon electrode for intracellular voltammetry. Anal. Chem. 1986, 58, 2088-2091. [CrossRef]

19. Pande, S.; Jana, S.; Sinha, A.K.; Sarkar, S.; Basu, M.; Pradhan, M.; Pal, A.; Chowdhury, J.; Pal, T. Dopamine molecules on Aucore-Agshell bimetallic nanocolloids: Fourier transform infrared, Raman, and surface-enhanced Raman spectroscopy study aided by density functional theory. J. Phys. Chem. C 2009, 113, 6989-7002. [CrossRef]

20. Palanisamy, S.; Yan, L.; Zhang, X.; He, T. Surface enhanced Raman scattering-active worm-like Ag clusters for sensitive and selective detection of dopamine. Anal. Methods 2015, 7, 3438-3447. [CrossRef]

21. Lee, N.-S.; Hsieh, Y.-Z.; Paisley, R.F.; Morris, M.D. Surface-Enhanced Raman Spectroscopy of the Catecholamine Neurotransmitters and Related Compounds. Anal. Chem. 1988, 60, 442-446. [CrossRef] [PubMed]

22. Menshykau, D.; Streeter, I.; Compton, R.G. Influence of Electrode Roughness on Cyclic Voltammetry. J. Phys. Chem. C 2008, 112, 14428-14438. [CrossRef]

23. Wan, Y.; Guo, Z.; Jiang, X.; Fang, K.; Lu, X.; Zhang, Y.; Gu, N. Quasi-spherical silver nanoparticles: Aqueous synthesis and size control by the seed-mediated Lee-Meisel method. J. Colloid Interface Sci. 2013, 394, $263-268$. [CrossRef] [PubMed]

24. Becke, A.D. Density-functional thermochemistry. III. The role of exact exchange. J. Chem. Phys. 1993, 98, 5648-5652. [CrossRef]

25. Lee, C.; Yang, W.; Parr, R.G. Development of the Colle-Salvetti correlation-energy formula into a functional of the electron density. Phys. Rev. B 1988, 37, 785-789. [CrossRef] 
26. Krishnakumar, V.; Keresztury, G.; Sundius, T.; Ramasamy, R. Simulation of IR and Raman spectra based on scaled DFT force fields: A case study of 2-(methylthio)benzonitrile, with emphasis on band assignment. J. Mol. Struct. 2004, 702, 9-21. [CrossRef]

27. Polavarapu, P.L. Ab initio vibrational Raman and Raman optical activity spectra. J. Phys. Chem. 1990, 94, 8106-8112. [CrossRef]

28. Keresztury, G. Handbook of Vibrational Spectroscopy; John Wiley \& Sons, Ltd.: Chichester, UK, 2001; Volume 1.

29. Pulay, P. Applications of Electronic Structure Theory; Plenum: New York, NY, USA, 1997; Volume 4.

30. Lin-Vien, D.; Colthup, N.B.; Fateley, W.G.; Grasselli, J.G. The Handbook of Infrared and Raman Characteristic Frequencies of Organic Molecules; Academic Press: San Diego, CA, USA, 1991.

31. Lambert, J.B.; Shurvell, H.F.; Cooks, R.G. Introduction to Organic Spectroscopy; Collier Macmillan Cop.: New York, NY, USA, 1987; pp. 174-177.

32. Mohammad-Shiri, H.; Ghaemi, M.; Riahi, S.; Akbari-Sehat, A. Computational and Electrochemical Studies on the Redox Reaction of Dopamine in Aqueous Solution. Int. J. Electrochem. Sci. 2011, 6, 317-336.

33. Ciubuc, J.D.; Qiu, C.; Bennet, K.E.; Alonzo, M.; Durrer, W.G.; Manciu, F.S. Raman computational and experimental studies of dopamine molecules on silver nanocolloids. In Proceedings of the 2017 IEEE International Symposium on Medical Measurements and Applications (MeMeA), Rochester, MN, USA, 7-10 May 2017; pp. 153-158. [CrossRef]

34. Yu, F.; Chen, S.; Chen, Y.; Li, H.; Yang, L.; Chen, Y.; Yin, Y. Experimental and theoretical analysis of polymerization reaction process on the polydopamine membranes and its corrosion protection properties for 304 Stainless Steel. J. Mol. Struct. 2010, 982, 152-161. [CrossRef]

(C) 2017 by the authors. Licensee MDPI, Basel, Switzerland. This article is an open access article distributed under the terms and conditions of the Creative Commons Attribution (CC BY) license (http:/ / creativecommons.org/licenses/by/4.0/). 